Review Article

\title{
Displacement Field Approximations for Force-Based Elements in Large Displacement Analyses
}

\author{
M. Vahidi, V. Jafari, M. H. Abyaneh, and SH. Vahdani \\ School of Civil Engineering, College of Engineering, University of Tehran, P.O. Box 11365-4563, \\ Tehran, Iran \\ Correspondence should be addressed to V. Jafari, vjafari@ut.ac.ir
}

Received 10 August 2011; Revised 24 February 2012; Accepted 15 March 2012

Academic Editor: Hester Bijl

Copyright (C) 2012 M. Vahidi et al. This is an open access article distributed under the Creative Commons Attribution License, which permits unrestricted use, distribution, and reproduction in any medium, provided the original work is properly cited.

\begin{abstract}
This paper investigates different approximation techniques for planar beam column elements in force-based methods. The three fields, introduced in this review, are: curvature-based displacement interpolation (CBDI) used in matrix-based flexibility formulations, linear displacement approximation applied in state space, and higher-order displacement approximation utilized again in state space. Using these three approximation fields, the responses and their accuracies in some systems are compared in examples. Finally, focusing on the accuracy and regarding the performed analyses, it seems that the computational cost is reduced and accuracy of responses is elevated in many engineering problems using the higher-order approximation field in state space.
\end{abstract}

\section{Introduction}

Traditionally, displacement-based methods are implemented in structural analyses. These methods have some drawbacks caused by their displacement field interpolations, particularly, in cases of high nonlinearities and nonprismatic beam sections. In this regard, equilibrium-based elements have been recently introduced to conquer the inefficient solution processes in displacement-based approaches.

There are a few researchers who have developed and worked on force-based techniques. Ciampi and Carlesimo [1] developed an equilibrium-based element for structural seismic analyses. Spacone et al. [2,3] as well as Petrangelti et al. [4] developed the mentioned element to more material nonlinearity effects in concrete elements.

Neuenhofer and Filippou [5] presented a force-based element for geometrically nonlinear analyses of plane frame structures; Taylor et al. [6] presented a mixed finite element method for beam-column problems. 
Generally, there are two main aspects of element state determinations, in equilibriumbased methods: the matrix-based approach, used primarily by Spacone et al. [2,3] and Taucer et al. [7]; the dynamical state space approach recently applied by Simeonov et al. [8] and then Sivaselvan and Reinhorn [9]. The former method is very common and straight forward in a finite element program. On the other hand, the later technique, called State Space Approach (SSA), is based on direct solution of governing differential Algebraic equations (DAEs) of a structural system. Therefore, the basic solution method differs from finite element formulations, which are developed based on structural matrix analyses.

It is noted that the accuracy of force-based elements is directly affected by their interelement displacement field approximation, which is used to evaluate the element nonlinear flexibility matrices. Three main approximation procedures have been proposed till now: displacement interpolation (CBDI) technique, linear field in state space approach, higher-order field in state-space approach. The first two approaches are introduced for EulerBernoulli straight beam-column elements; however, the third one is capable of analyzing Timoshenko curved frame elements.

The first technique is primarily developed by Neuenhofer and Filippou [5] for a geometrical nonlinear Euler-Bernoulli force-based beam-column element. Magalhaesde Souza [10] extended this formulation to inelastic spatial frame analyses. Lagrange polynomials are used in their technique to interpolate curvatures at integration points for displacement field approximations. Because of the fact that rotations are assumed small in this procedure, large deformation problems need more elements in this technique.

The second one was at first proposed by Sivaselvan and Reinhorn [9]. According to this technique, a linear interpolation is used to evaluate the effects of large internal rotations. Although building structural systems without high nonlinearities are effectively analyzed by their approach, no reliable responses are obtained in very large displacements and deformations.

The third approach is presented by Jafari et al. [11] and offers a higher-order displacement field approximation to be applied to the governing differential algebraic equations upon Lagrange polynomials in state space. The procedure is applied to planar elastic Timoshenko curved beams considering large displacements and interelement rotations.

This paper reviews firstly the basic concepts of a general Timoshenko forcebased planar curved element. Afterwards, three mentioned approximation procedures are explained. A brief comparison is performed using different approximation procedures applied for equilibrium-based elements till now. Finally, the numerical examples compare the results obtained by these different approximation techniques.

\section{State Equations}

An initially curved frame is considered without any internal loading. The end displacement and force vector components of this element are shown in Figure 1(a) in the global planar coordinate system. The adopted local coordinate system is shown in Figure 1(b) based on the corotational transformation technique. Three independent local degrees of freedom, demonstrated in Figure 1(b), are

$$
\begin{aligned}
& \mathbf{p}=\left\langle\begin{array}{lll}
P_{1} & P_{2} & P_{3}
\end{array}\right\rangle^{T}=\left\langle\begin{array}{lll}
N & M_{I} & M_{J}
\end{array}\right\rangle^{T} \\
& \boldsymbol{\delta}=\left\langle\begin{array}{lll}
D_{1} & D_{2} & D_{3}
\end{array}\right\rangle^{T}=\left\langle\begin{array}{lll}
u_{I} & \theta_{I} & \theta_{J}
\end{array}\right\rangle^{T},
\end{aligned}
$$




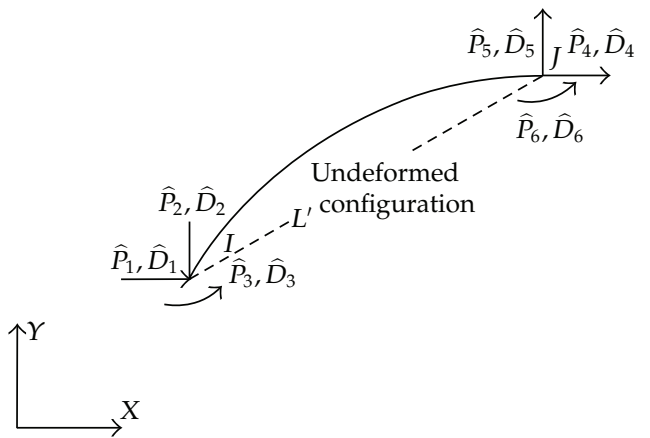

(a)

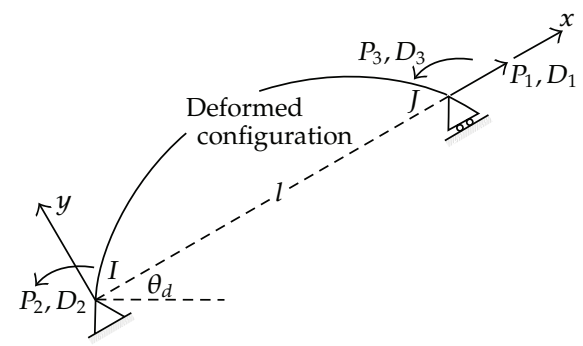

(b)

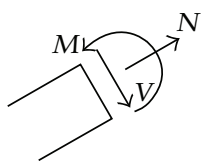

(c)

Figure 1: (a) Global coordinate system and degrees of freedom for a planar undeformed curved element. (b) Local adopted coordinate system without rigid body modes in element deformed state. (c) Force vector components on a sample section.

where $N=$ axial load; $M_{I}$ and $M_{J}=$ two corotational moments; $u_{I}=$ axial displacement; $\theta_{I}$ and $\theta_{J}=$ two end rotations.

In order to evaluate the element flexibility matrix, the equilibrium and compatibility equations should be presented for curved beams. The Reissner's kinematical exact stress resultant theory [12] is applied for curved beams to obtain equilibrium equations for geometrical nonlinear beam with end forces by Jafari et al. [13, 14]. Hence, we have the following

\section{(a) Equilibrium Equations}

One has

$$
\mathbf{s}\left(s_{0}\right)=\left[\begin{array}{c}
N\left(s_{0}\right) \\
M\left(s_{0}\right) \\
V\left(s_{0}\right)
\end{array}\right]=\boldsymbol{\Gamma}\left(s_{0}\right)\left[\begin{array}{l}
P_{1} \\
P_{2} \\
P_{3}
\end{array}\right]=\Gamma\left(s_{0}\right) \mathbf{p},
$$

where

$$
\Gamma(s)=\left[\begin{array}{ccc}
\cos \left(\theta\left(s_{0}\right)\right) & -\sin \left(\theta\left(s_{0}\right)\right) / l & -\sin \left(\theta\left(s_{0}\right)\right) / l \\
\eta & \xi / l-1 & \xi / l \\
-\sin \left(\theta\left(s_{0}\right)\right) & -\cos \left(\theta\left(s_{0}\right)\right) / l & -\cos \left(\theta\left(s_{0}\right)\right) / l
\end{array}\right]
$$

and $l=L+D_{1}$, shown in Figure 1(b). 
Here, a special point is assumed with local coordinates of $(x, y)$ on the element centre line moving to $(\xi, \eta)$ in the deformed configuration.

$\theta$ is angle between beam cross-section and $y$-axis;

$\mathbf{s}\left(s_{0}\right)$ is vector of arbitrary section force components, Figure $1(\mathrm{c}) ; N\left(s_{0}\right)$ is axial force; $M\left(s_{0}\right)$ is moment; $V\left(s_{0}\right)$ is shear.

\section{(b) Compatibility Equations}

Compatibility equations in state space are:

Finally, the matrix form of compatibility equation is:

$$
\dot{\boldsymbol{\delta}}=\int_{s_{0}} \Gamma^{\mathrm{T}} \dot{\mathbf{d}}\left(s_{0}\right) d s_{0}
$$

where $\mathbf{d}\left(s_{0}\right)=$ vector of generalized strains as

$$
\mathbf{d}\left(s_{0}\right)=\left\langle\varepsilon_{0}\left(s_{0}\right) \theta^{\prime}\left(s_{0}\right)-\theta_{0}^{\prime}\left(s_{0}\right) \gamma\left(s_{0}\right)\right\rangle^{T}
$$

(c) Element State Equations

The element state equation is

$$
\left(\int_{s_{0}}\left[\boldsymbol{\Gamma}\left(s_{0}\right)^{T} \boldsymbol{\Phi}\left(s_{0}\right) \boldsymbol{\Gamma}\left(s_{0}\right)\right] d s_{0}\right) \dot{\mathbf{p}}=\dot{\boldsymbol{\delta}}-\left(\int_{s_{0}}\left[\boldsymbol{\Gamma}\left(s_{0}\right)^{T} \boldsymbol{\Phi}\left(s_{0}\right) \dot{\boldsymbol{\Gamma}}\left(s_{0}\right)\right] d s_{0}\right) \mathbf{p},
$$

where

$$
\boldsymbol{\Phi}\left(s_{0}\right)=\left[\begin{array}{ccc}
E_{T} A & 0 & 0 \\
0 & E_{T} I & 0 \\
0 & 0 & G_{T} A_{s}
\end{array}\right]^{-1},
$$

where $\mathbf{\Phi}\left(s_{0}\right)$ is section flexibility matrix; $E_{T}$ is axial material tangent modulus; $G_{T}$ is shear material tangent modulus; $A$ is section area; $I$ is inertia; $A_{s}$ is shear area.

\section{Displacement Field Approximations in Force-Based Methods}

An interelement displacement field is required to evaluate the flexibility matrices in (2.6) matrix for geometrical nonlinear state. Besides, the element accuracy is extensively related to displacements approximation procedures. So, the existed techniques are explained hereafter.

\subsection{CBDI}

Neuenhofer and Filippou developed a geometrically force-based element in matrix-based formulation. The flexibility matrix in their formulas is a simple form of (2.6) and requires 
a field approximation; it consists of displacement terms. It is noted that a straight EulerBernoulli beam is considered in this formulation. They named their technique, curvaturebased displacement interpolation, "CBDI."

Here, transverse displacements are required in the flexibility matrix, and rotations are assumed small. So, CBDI technique is used only for evaluating the transverse displacement field. This field is obtained using curvatures at integration points for planar case by

$$
\begin{aligned}
& \mathbf{v}=\mathbf{L} \boldsymbol{\kappa} \\
& \mathbf{L}=L^{2}\left[\begin{array}{cccc}
\frac{1}{2}\left(X_{1}^{2}-X_{1}\right) & \frac{1}{6}\left(X_{1}^{3}-X_{1}\right) & \cdots & \frac{1}{N G(N G+1)}\left(X_{1}^{N G+1}-X_{1}\right) \\
\vdots & \vdots & \ddots & \vdots \\
\frac{1}{2}\left(X_{N G}^{2}-X_{N G}\right) & \frac{1}{6}\left(X_{N G}^{3}-X_{N G}\right) & \cdots & \frac{1}{N G(N G+1)}\left(X_{N G}^{N G+1}-X_{N G}\right)
\end{array}\right] \mathbf{G}^{-1}, \\
& \mathbf{G}=\left[\begin{array}{ccccc}
1 & X_{1} & X_{1}^{2} & \ldots & X_{1}^{\mathrm{NG}-1} \\
\vdots & \vdots & \vdots & & \ddots \\
1 & X_{\mathrm{NG}} & X_{\mathrm{NG}}^{2} & \cdots & X_{\mathrm{NG}}^{\mathrm{NG}-1}
\end{array}\right]
\end{aligned}
$$

where $\mathbf{v}$ is transverse displacement field vector; $\mathbf{L}$ is influence matrix including polynomials; $\mathrm{G}$ is so called Vandermnode matrix; $X_{i}$ is coordinate of integration point $i$ in the local system; $i$ is changes from 1 to NG; NG is Number of integration points; $\boldsymbol{\kappa}$ is curvature vector at integration points.

Although this approximation performs well in many analyses, it lacks in accuracy where large internal rotations happen in an analysis. This is because of the fact that the rotations have been neglected in this formulation.

Jafari et al. [14] extended CBDI to curvature/shearing-based displacement interpolation (CSBDI) to capture shear effects in force-based elements.

\subsection{Linear Displacement Field Approximation in State Space}

As it is seen in (2.6), the displacement field includes $\xi, \eta$, and $\theta$ for the element. Using a rate form equation based on the strain displacement relationship (Huddleston [15]), a linear approximation procedure was proposed by Sivaselvan and Reinhorn [9] in their analyses.

As mentioned in matrix-based formulation, shear effects as well as initial curvature effects are not considered in this method. However, its extension to shear deformable curved element is very straightforward as described in Section 2. The strain-displacement relations presented by Huddleston [15] are

$$
\begin{aligned}
& \frac{d \xi}{d x}=\left(1+\varepsilon_{0}(x)\right) \cos (\theta(x)), \\
& \frac{d \eta}{d x}=\left(1+\varepsilon_{0}(x)\right) \sin (\theta(x)) .
\end{aligned}
$$


In this procedure, the deformations and local coordinates are monitored at NG integration points in terms of NG parameters as

$$
\theta_{j}^{\prime}=\boldsymbol{\theta}^{\prime}\left(x_{j}\right), \quad \varepsilon_{j}=\varepsilon_{0}\left(x_{j}\right), \quad \xi_{i}=\boldsymbol{\xi}\left(x_{j}\right), \quad \eta_{i}=\boldsymbol{\eta}\left(x_{j}\right),
$$

where $\boldsymbol{\theta}^{\prime}\left(x_{j}\right)$ is the $j$ th element of rotation derivative vector; $\varepsilon_{0}\left(x_{j}\right)$ is the $j$ th element of axial strain vector; $\xi\left(x_{j}\right)$ is the $j$ th element of $\xi$ local coordinates vector; $\boldsymbol{\eta}\left(x_{j}\right)$ is the $j$ th of $\eta$ local coordinates vector.

Here, an implicit first-order method is used to integrate (3.2) as initial value problems (IVP), for two times from two ends. Eventually, a weighted average is taken for approximation. For this purpose the formulas are mentioned bellow, starting from one end of the element

$$
\begin{gathered}
\theta_{i+1}=\theta_{i}+\left(X_{i+1}-X_{i}\right) \theta_{i+1}^{\prime} \\
\xi_{i+1}=\xi_{i}+\left(X_{i+1}-X_{i}\right)\left(1+\varepsilon_{i+1}\right) \cos \left(\theta_{i+1}\right), \\
\eta_{i+1}=\eta_{i}+\left(X_{i+1}-X_{i}\right)\left(1+\varepsilon_{i+1}\right) \sin \left(\theta_{i+1}\right) .
\end{gathered}
$$

This time, the equations are integrated as IVPs starting from the other end of element. Finally, displacement fields are evaluated at every integration points using weighted average of two solutions.

Due to the fact that this formulation counts for large internal rotations, its accuracy in analyses with large deformations is well elevated in comparison with pervious formulations. Therefore, a linear field in approximation can result in acceptable responses just in building structures where not very high geometric nonlinearities happen. In order to improve this accuracy and obtain reliable results, a large number of integration points and/or more elements are required. Consequently, the computational cost is considerably increased.

In addition, using Huddleston strain-displacement relationship leads to nonlinear rotational terms, and consequently more accurate results are predicted. However, the accuracy is obviously weakened applying this technique; that is, how to approximate the field is of high importance. The deficiency of linear approximation is confirmed in many examples in comparison with CBDI and CSBDI (Jafari et al. [14]).

\subsection{Higher-Order Displacement Field Approximation in State Space}

As mentioned previously, the displacement field components $\xi, \eta$, and $\theta$ need to be evaluated at integration points. Here, a higher-order displacement approximation method is adjusted using Lagrange polynomials.

The method proposed by Jafari et al. [11] is mainly composed of two techniques, linear approximation and CBDI. In this formulation, again the deformations are monitored at NG integration points in terms of NG parameters as

$$
\theta_{j}^{\prime}=\boldsymbol{\theta}^{\prime}\left(s_{j}\right), \quad \gamma_{j}=r\left(s_{j}\right), \quad \varepsilon_{j}=\boldsymbol{\varepsilon}_{0}\left(s_{j}\right), \quad \xi_{i}=\boldsymbol{\xi}\left(s_{j}\right), \quad \eta_{i}=\boldsymbol{\eta}\left(s_{j}\right),
$$

where $s_{j}$ is local coordinates of integration points on curved beam; $\boldsymbol{\gamma}\left(s_{j}\right)$ is the $j$ th element of shear strain vector. 
According to above assumption, the bellow formulas can be written as

$$
\begin{aligned}
& \varepsilon(s)=\sum_{j=1}^{N G} l_{j}(s) \varepsilon_{j}, \\
& \theta^{\prime}(s)=\sum_{j=1}^{N G} l_{j}(s) \theta_{j}^{\prime}, \\
& \gamma(s)=\sum_{j=1}^{N G} l_{j}(s) \gamma_{j},
\end{aligned}
$$

where, $l_{j}(s)$ is Lagrangian polynomials, obtained from (3.7).

We have

$$
l_{j}(s)=\frac{\prod_{i=1, i \neq j}^{\mathrm{NG}}\left(s-s_{0 i}\right)}{\prod_{i=1, i \neq j}^{\mathrm{NG}}\left(s_{0 j}-s_{0 i}\right)},
$$

thus,

$$
l_{j}\left(s_{i}\right)=\delta_{i j}
$$

Regarding the necessity of integrating over functions, the more applicable form of Lagrange polynomials is used for closed form integration as

$$
\left[\begin{array}{llll}
l_{1}(s) & l_{2}(s) & \ldots & l_{\mathrm{NG}}(s)
\end{array}\right]=\left[\begin{array}{llll}
1 & s & \ldots & s^{\mathrm{NG}-1}
\end{array}\right] \mathrm{G}^{-1} .
$$

This matrix is determined only one time as the number of integration points remains constant during analysis. Starting from one end of the element, the incremental form of internal displacement field components can be stated as

$$
\begin{aligned}
& \theta_{i+1}=\theta_{i}+\Delta \theta_{i, i+1}, \\
& \xi_{i+1}=\xi_{i}+\Delta \xi_{i, i+1}, \\
& \eta_{i+1}=\eta_{i}+\Delta \eta_{i, i+1} .
\end{aligned}
$$

The incremental values are written, using a higher-order and more accurate integration, as

$$
\begin{gathered}
\Delta \theta_{i j}=s_{0}\left\langle s \frac{s^{2}}{2} \frac{s^{2}}{3} \cdots \frac{s^{\mathrm{NG}}}{\mathrm{NG}}\right\rangle \mathbf{G}^{-1} \boldsymbol{\theta}^{\prime} \mid \begin{array}{l}
s=s_{j} \\
s=s_{i},
\end{array} \\
\Delta \xi_{i j}=\int_{s_{i}}^{s_{j}}\left(\left(1+\varepsilon_{0}\left(s_{0}\right)\right) \cos \left(\theta\left(s_{0}\right)\right)-r \sin \left(\theta\left(s_{0}\right)\right)\right) d s_{0}, \\
\Delta \eta_{i j}=\int_{s_{i}}^{s_{j}}\left(\left(1+\varepsilon_{0}\left(s_{0}\right)\right) \sin \left(\theta\left(s_{0}\right)\right)+\gamma\left(s_{0}\right) \cos \left(\theta\left(s_{0}\right)\right)\right) d s_{0} .
\end{gathered}
$$




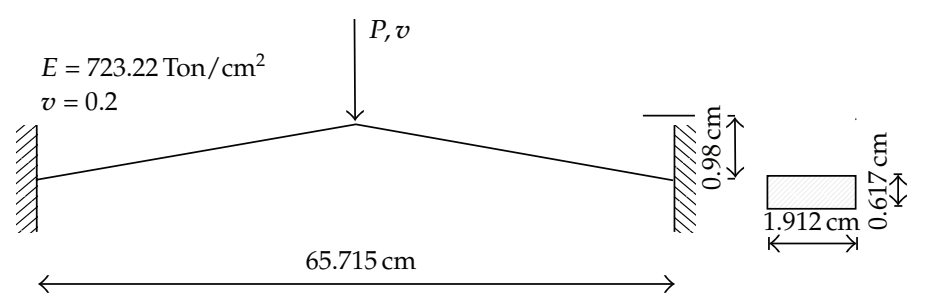

Figure 2: Williams toggle frame.

These integrations are achieved based on the local Gauss or Gauss-Lobatto procedure performed between $s=s_{i}$ and $s=s_{j}$.

In this way, the displacement field is obtained from one end of the element. The same procedure is repeated from other end performing the operations as (3.10) and (3.15). Finally, the weighted averages of these two approximations are evaluated, and three displacement field components are obtained. In this study, the weights are considered the same for $\xi, \eta$, and $\theta$, in every integration points.

As it is demonstrated next, the results from this approximation technique are more accurate in comparison with those of other procedures. Besides, the shear and curvilinear effects are considered in the analyses.

It is seen from different analyses that there is no significant change in computational cost using this method, in comparison with the previous ones. Regarding the former, Lagrangian polynomials are used in both CBDI method and the one presented here. However, large deformations, including shear and curvilinear effects, are considered by this new method, and, consequently, more accurate results are obtained.

In linear approximation, despite considering large deformations for Euler- Bernoulli beam, a large number of integration points are needed in order to meet accurate results. The computational cost is increased significantly as the consequence of this phenomenon.

\section{Numerical Examples}

\subsection{Williams Toggle Frame (Figure 2)}

This example is to confirm the accuracy of the results obtained by this new displacement field approximation in comparison with linear field one. Toggle frame has analyzed elastically by several researchers for surveying geometrical nonlinear formulations; the frame and its properties are shown in Figure 3.

In Figure 4, the equilibrium curve is evaluated using one element per half of the structure with four Lobatto points inside. The results, presented by the formulation with higher-order approximation, are more reliable and more efficient comparing with linear method in force-based technique. It should be noted that ten Lobatto points per element are used in linear approximation.

The results of CBDI technique are approximately similar to those of the proposed method as large internal rotations are not included in the problem. As it is seen in Figure 3, the accuracy is not significantly changed by increasing the number of internal integration points; therefore, four Lobatto points seem enough. 


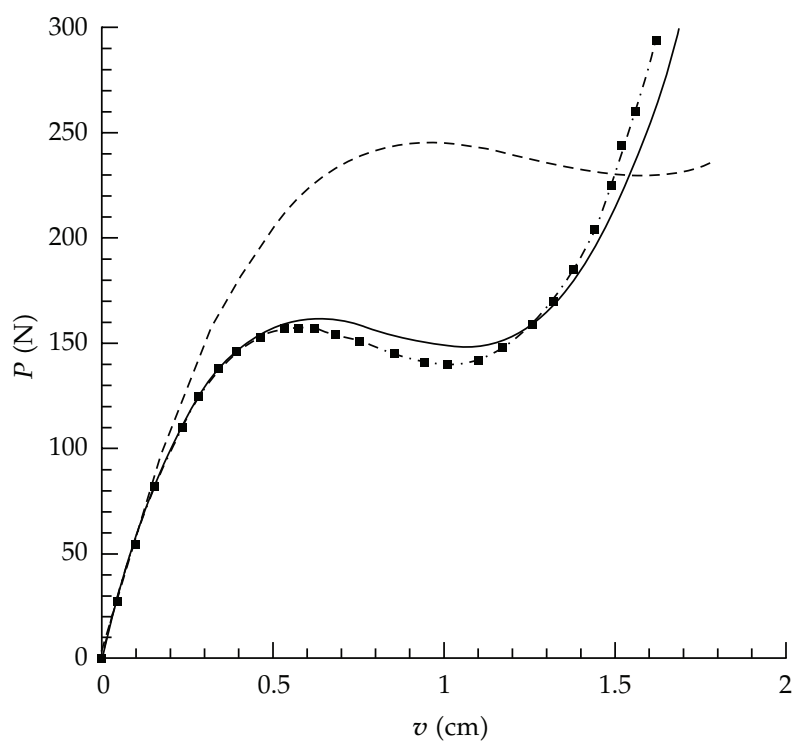

$\rightarrow-$ Williams (analytical)

- - - State-space approach, linear approximation field, 1 elem.

- State-space approach, proposed approximation field, 1 elem.

Figure 3: The equilibrium paths of toggle frame from: (i) analytical solution; (ii) state space with linear approximation, 1 element per half of the structure; 10 Lobatto points; (iii) state space with higher-order approximation method, 1 element per half of the structure; 4 Lobatto points.

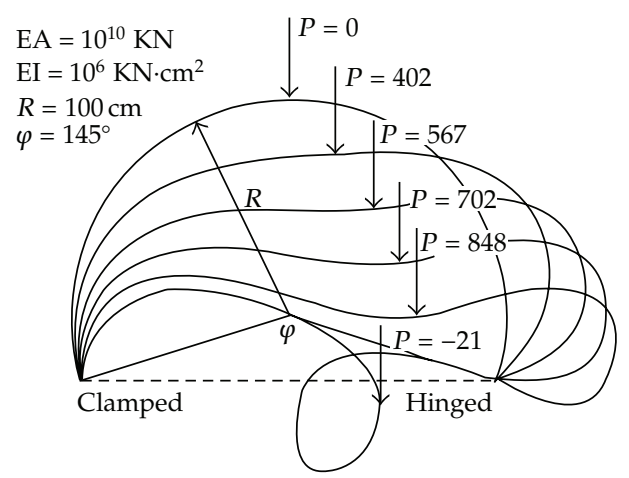

Figure 4: Circular arc geometry and deformed shape in some different load levels.

\subsection{Postbuckling Analysis of a Clamped-Hinged Circular Arch}

This well-known example presents the geometric nonlinear analysis of the pre- and Postbuckling deformation of a circular arch, hinged at one end and clamped at the other end, under a vertical force applied at the apex. This problem was suggested by da Deppo and Schmidt [16] and has been analyzed by many researchers. (Simo and Vu-Quoc [17]; Ibrahimbegovic, and Frey [18]). 


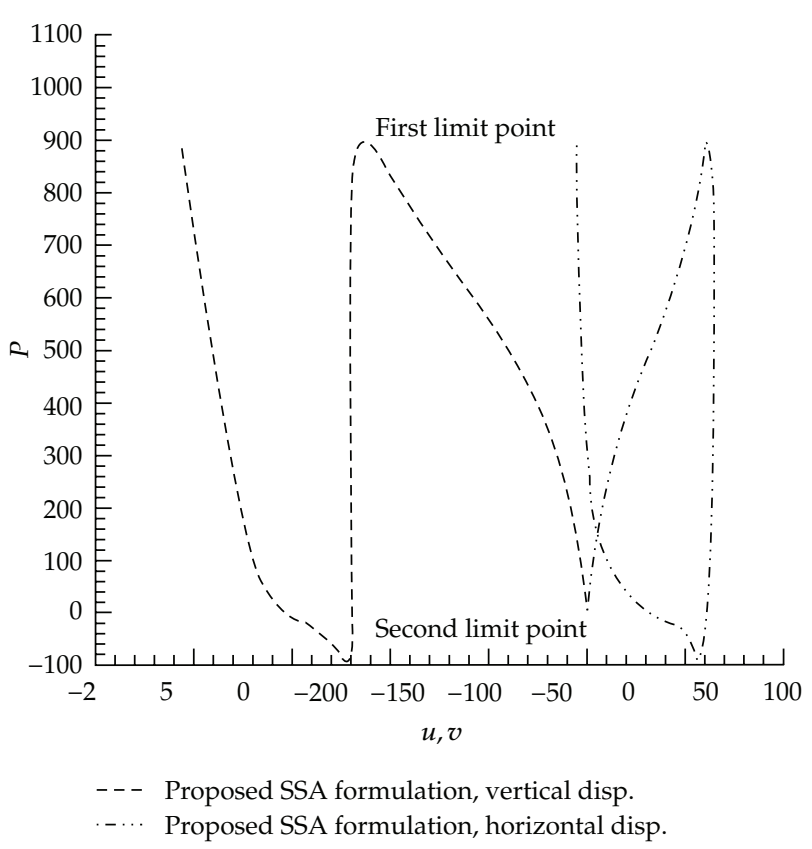

Figure 5: Postbuckling behavior of the circular arc, using higher order approximation field.

Material and geometric data for the arch are shown in Figure 4. As responses show, the problem includes a sharp negative slope after the first buckling load is reached. The use of higher-order approximation field as well as continuous form of constraint equation results in robust responses as are demonstrated in Figure 5 for eight elements and five Lobatto points.

The use of arc length solution technique assists to trace the complete equilibrium path which includes a steep downward slope. For this problem, ten elements with five internal Lobatto points result in accurate responses.

\section{Conclusions}

Three main approximation fields are reviewed: CBDI in matrix-based formulations, linear approximation in state space, and Lagrange polynomial-based displacement field approximation for curved elements in state space. The later field is basically resulted from two former methods. While fewer elements and integration points are used in the latter method, the results gained for high nonlinear problems are more accurate and reliable. Besides, the efficiency of presented method is not affected, comparing to linear approximation procedure, because of the fact that applying lower number of integration points results in reliably accurate responses. The approximating polynomials are not generally high ordered; therefore, nonphysical wriggling is avoided. However, it seems that using an approximation like Bsplines may enhance the efficiency even better.

\section{References}

[1] V. Ciampi and L. Carlesimo, "A nonlinear beam element for seismic analysis of structures," in Proceedings of the European Conference on Earthquake Engineering, pp. 73-80, Lisbon, Portugal, September 1986. 
[2] E. Spacone, V. Ciampi, and F. C. Filippou, "Mixed formulation of nonlinear beam finite element," Computers and Structures, vol. 58, no. 1, pp. 71-83, 1996.

[3] E. Spacone, F. C. Filippou, and F. F. Taucer, "Fibre beam-column model for non-linear analysis of R/C frames: part I. Formulation," Earthquake Engineering and Structural Dynamics, vol. 25, no. 7, pp. 711-725, 1996.

[4] M. Petrangeli, P. E. Pinto, and V. Ciampi, "Fiber element for cyclic bending and shear of RC structures. I: theory," Journal of Engineering Mechanics, vol. 125, no. 9, pp. 994-1001, 1999.

[5] A. Neuenhofer and F. C. Filippou, "Geometrically nonlinear flexibility-based frame finite element," Journal of Structural Engineering, vol. 124, no. 6, pp. 704-711, 1998.

[6] R. L. Taylor, F. C. Filippou, A. Saritas, and F. Auricchio, "A mixed finite element method for beam and frame problems," Computational Mechanics, vol. 31, no. 1-2, pp. 192-203, 2003.

[7] F. Taucer, E. Spacone, and F. C. Filippou, "A fiber beam-column element for seismic response analysis of reinforced concrete structures," EERC report, Earthquake Engineering Research, 1992.

[8] V. K. Simeonov, M. V. Sivaselvan, and A. M. Reinhorn, "Nonlinear analysis of structural frame systems by the state-space approach," Computer-Aided Civil and Infrastructure Engineering, vol. 15, no. 2, pp. 76-89, 2000.

[9] M. V. Sivaselvan and A. M. Reinhorn, "Collapse analysis: large inelastic deformations analysis of planar frames," Journal of Structural Engineering, vol. 128, no. 12, pp. 1575-1583, 2002.

[10] R. Magalhaes de Souza, Force-based finite element for large displacement inelastic analysis of frames, $\mathrm{PhD}$ dissertation, Department of Civil and Environmental Engineering, University of California, Berkeley, Calif, USA, 2000.

[11] V. Jafari, M. A. Abyaneh, S. H. Vahdani, and M. Rahimian, "Improved displacement-field approximation for geometrical nonlinear flexibility-based planar curved element in state space," Mechanics Based Design of Structures and Machines, vol. 37, no. 4, pp. 475-502, 2009.

[12] E. Reissner, "On finite deformations of space curved beams," Journal of Applied Mathematics and Physics, vol. 32, no. 6, pp. 734-744, 1981.

[13] V. Jafari, M. Rahimian, and S. H. Vahdani, "Improved formulation in mixed-based state-space approach for large displacement inelastic analysis of frames," Journal of Engineering Mechanics, vol. 137, no. 3, pp. 196-205, 2011.

[14] V. Jafari, S. H. Vahdani, and M. Rahimian, "Derivation of the consistent flexibility matrix for geometrically nonlinear Timoshenko frame finite element," Finite Elements in Analysis and Design, vol. 46, no. 12, pp. 1077-1085, 2010.

[15] J. V. Huddleston, "A nonlinear analysis of elastically constrained tie-rods under transverse loads," International Journal of Mechanical Sciences, vol. 21, no. 10, pp. 623-630, 1979.

[16] D. A. da Deppo and R. Schmidt, "Instability of clamped-hinged circular arches subjected to a point load," Journal of Applied Mechanics, vol. 97, no. 4, pp. 894-896, 1975.

[17] J. C. Simo and L. Vu-Quoc, "A three-dimensional finite-strain rod model. part II: computational aspects," Computer Methods in Applied Mechanics and Engineering, vol. 58, no. 1, pp. 79-116, 1986.

[18] A. Ibrahimbegovic and F. Frey, "Finite element analysis of linear and non-linear planar deformations of elastic initially curved beams," International Journal for Numerical Methods in Engineering, vol. 36, no. 19, pp. 3239-3258, 1993. 


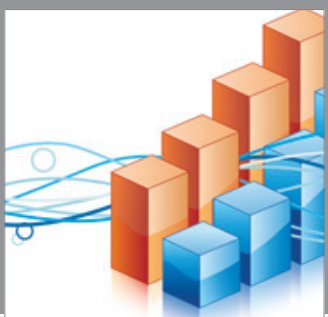

Advances in

Operations Research

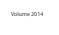

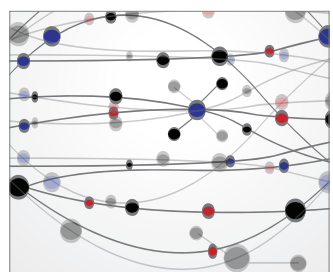

\section{The Scientific} World Journal
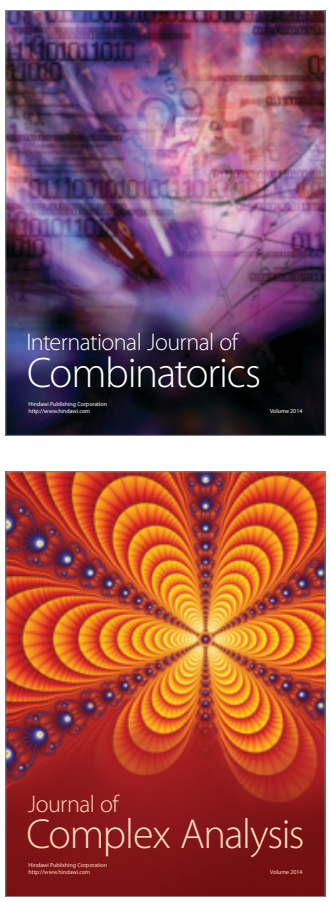

International Journal of

Mathematics and

Mathematical

Sciences
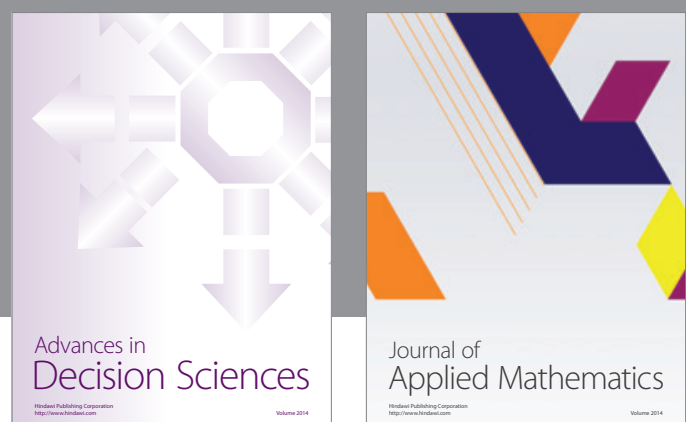

Journal of

Applied Mathematics
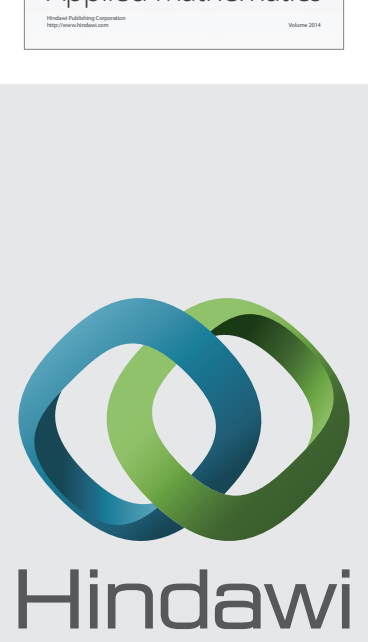

Submit your manuscripts at http://www.hindawi.com
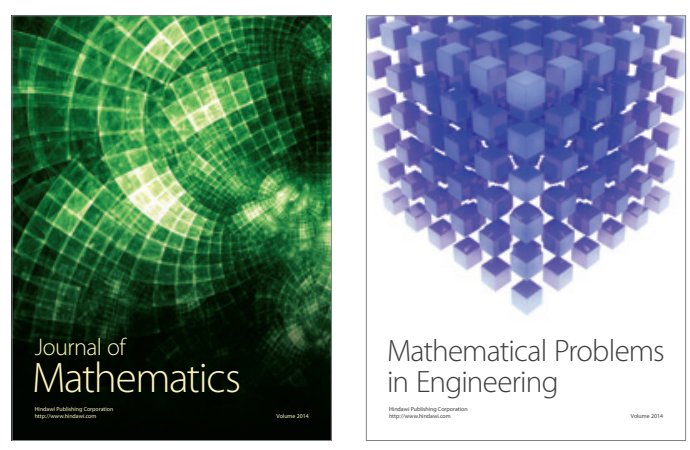

Mathematical Problems in Engineering
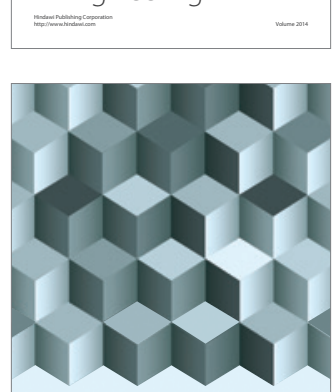

Journal of

Function Spaces
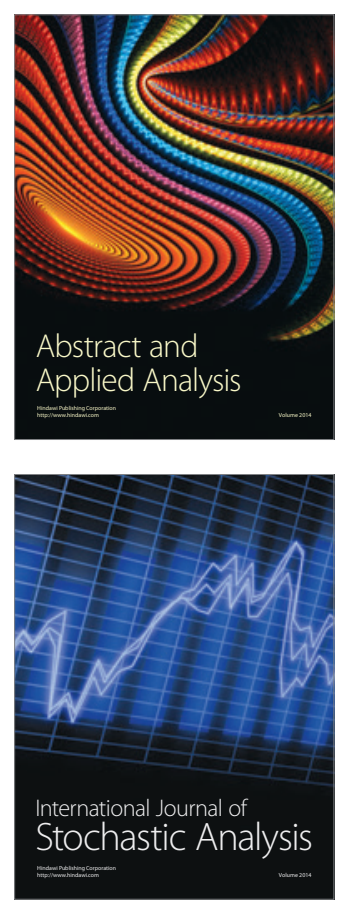

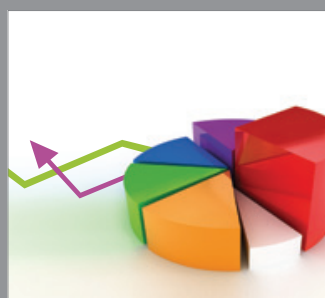

ournal of

Probability and Statistics

Promensencen
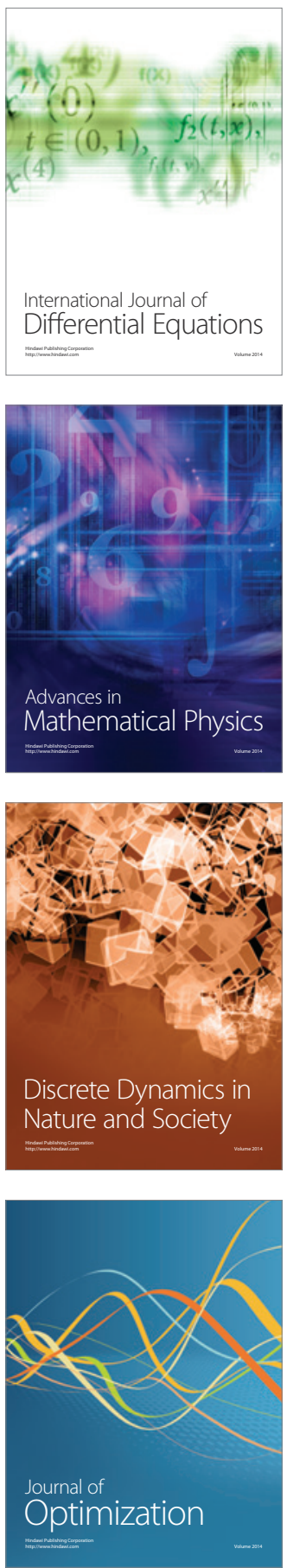